\title{
Mode referencing of an external cavity diode laser for continuous frequency stabilization
}

\author{
David Mclnerney \\ Michael Lynch \\ John F. Donegan \\ Vincent Weldon \\ Trinity College Dublin \\ School of Physics \\ Semiconductor Photonics Group \\ Dublin 2, Dublin D2 \\ Ireland \\ E-mail: mcinerneydavid@yahoo.ie
}

\begin{abstract}
The output of an external cavity diode laser (ECDL) is frequency stabilized to a water $\left(\mathrm{H}_{2} \mathrm{O}\right)$ vapor absorption line at $935 \mathrm{~nm}$ using wavelength modulation spectroscopy (WMS) by means of a dualfeedback locking loop. A novel mode-referencing technique is presented that, in conjunction with the feedback locking mechanism, helps improve the overall frequency-locking system. The mode-referencing enables the device output to maintain its locked frequency in the presence of environmental and other effects, which may cause the operational characteristics of the laser to drift. (C) 2008 Society of Photo-Optical Instrumentation Engineers. [DOI: 10.1117/1.2844700]
\end{abstract}

Subject terms: mode-referencing; external cavity diode laser; frequency stabilization.

Paper 070021R received Jan. 9, 2007; revised manuscript received Oct. 1, 2007; accepted for publication Oct. 17, 2007; published online Mar. 3, 2008.

\section{Introduction}

The ability to stabilize the emission from a diode laser to a reference frequency is of great importance for many applications including optical telecommunications, injection locking of high-power lasers, and spectroscopic based studies. ${ }^{1-4}$ However, depending on the type of device involved, control of the frequency-stabilizing mechanism may vary in complexity. For example, some widely tuneable sources with multisection architectures, such as the sampled-grating distributed Bragg reflector (SG-DBR) laser, require several current sources to drive the device, and very precise manipulation of these is required to control and stabilize the output emission wavelength. ${ }^{5}$ This situation is similar to that pertaining to the external cavity diode laser (ECDL), where the emission wavelength is controlled by both injection current to the laser and a voltage to a piezoelectric transducer (PZT), which controls the position of the external grating. ${ }^{6}$ ECDLs have long been a source of great interest due to their wide wavelength tuning capability, high output power, and, of course, the narrow linewidth available with an external cavity. ${ }^{7,8}$

To operate the ECDL such that the wavelength is tuned in a continuous, mode-hop-free manner, which is essential for successful frequency stabilization, a tuning contour plane was first recorded and is shown in Fig. 1. This is achieved by changing both current and PZT voltage simultaneously over a certain range and measuring the output power from the laser. Once a picture of the ECDL emission mode profile is obtained in this manner, a series of points are chosen along a region of the contour map, and these points are fitted with a polynomial. By simultaneously tuning the injection current and PZT voltage values, determined by the polynomial fit, the desired wavelength region can be scanned in a continuous linear fashion, as indicated by the dashed arrow in Fig. 1. In general, for most widely tuneable laser diodes, a similar situation pertains in that a

0091-3286/2008/\$25.00 @ 2008 SPIE mode profile of the output emission is first obtained so that correct operating currents can be chosen for continuous wavelength tuning of the device. ${ }^{9}$ Of course, it is assumed that the mode profile initially recorded remains constant with time so that the device can operate in a continuous wavelength tuning mode without fail using the determined control parameters. Unfortunately, this is not the case for some widely tuneable laser diodes ${ }^{10,11}$ and for the ECDL used in this work.

A shift in the tuning contour plane was observed over time caused by thermal or mechanical instabilities in the complex design of the laser. ${ }^{12}$ Obviously, this has a deleterious knock-on effect if the device is used in a frequency stabilization scheme, where a loss in continuous wavelength tuning ultimately leads to a complete failure in the frequency stabilization of the laser output. To counteract

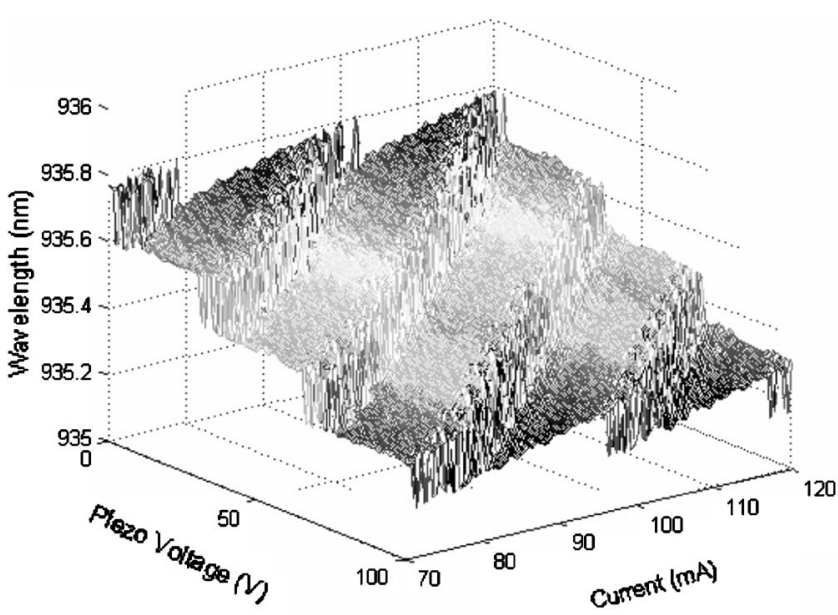

Fig. 1 Contour map of ECDL emission wavelength as a function of current and PZT voltage at a constant temperature of $20^{\circ} \mathrm{C}$ with the continuous wavelength tuning path clearly indicated with a dashed arrow. 


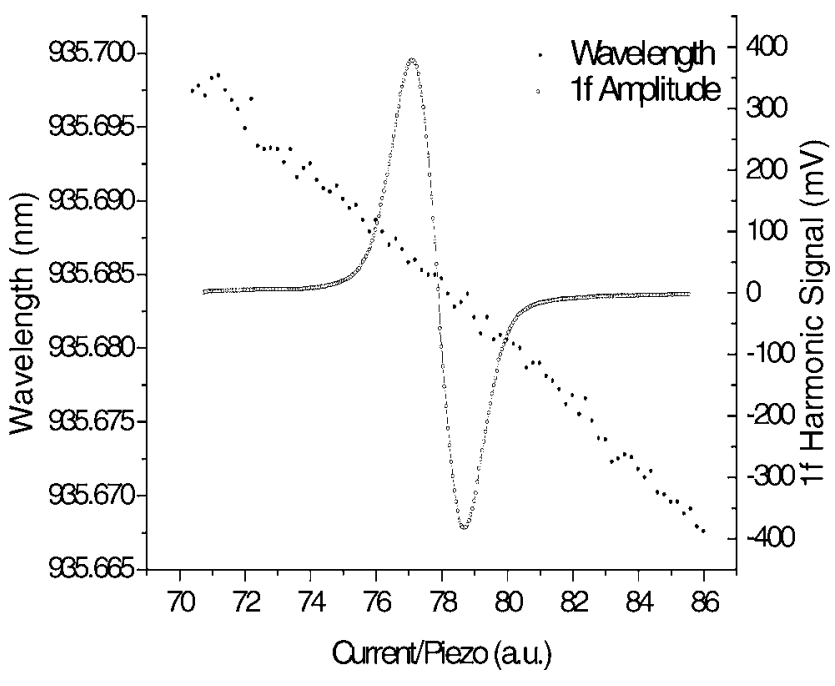

Fig. 2 Measurement of the $1 f$ harmonic signal of a detected water vapor absorption line at $935.684 \mathrm{~nm}$ used to lock the frequency of the device and the continuous wavelength tuning operation of the ECDL.

this problem, a mode-referencing technique was implemented into the frequency stabilization scheme similar in concept to a form of mode stabilization for a superstructure grating DBR (SSG-DBR) laser. ${ }^{13}$ Referencing (adjustment of operation parameters) of the ECDL (while maintaining frequency lock) ensures that the constant frequency emission remains on a characteristic line in the power plane equidistant from adjacent mode boundaries, which corresponds to the optimum operating point. With the modereferencing step, it was possible to achieve in situ monitoring of the position of the current/PZT voltage combination tuning path and compensate for any drift in the mode profile by locking the wavelength-tuning path to the center of the desired lasing mode. We demonstrate experimentally that it is possible to maintain frequency stabilization of an ECDL output to a $\mathrm{H}_{2} \mathrm{O}$ absorption line with the inclusion of the mode-referencing technique in the frequency-locking system, while applying a series of induced temperature steps, simulating thermal instability, to the device heat sink.

\section{Experimental Details}

An ECDL operating in the Littrow architecture was frequency stabilized to a $\mathrm{H}_{2} \mathrm{O}$ absorption line at $935.684 \mathrm{~nm}$ using wavelength modulation spectroscopy. ${ }^{14,15}$ (WMS). The spectral characteristics of the ECDL were initially measured and the device showed good single-mode emission at room temperature with a side-mode suppression ratio (SMSR) $>40 \mathrm{~dB}$ and the spectral linewidth of the device was $<300 \mathrm{kHz}$, measured using a short-arm homodyne technique. The maximum continuous mode-hopfree wavelength tuning of the laser, over the full range of the injection current and the PZT voltage, was measured to be approximately $0.45 \mathrm{~nm}$ and the maximum output power was $>60 \mathrm{~mW}$. Figure 2 shows the $1 f$ demodulated signal of the $\mathrm{H}_{2} \mathrm{O}$ absorption line with the zero-crossing point at the line center used to frequency lock the ECDL along with the continuous wavelength tuning of the laser output. In any frequency stabilization system, it is necessary to gen-

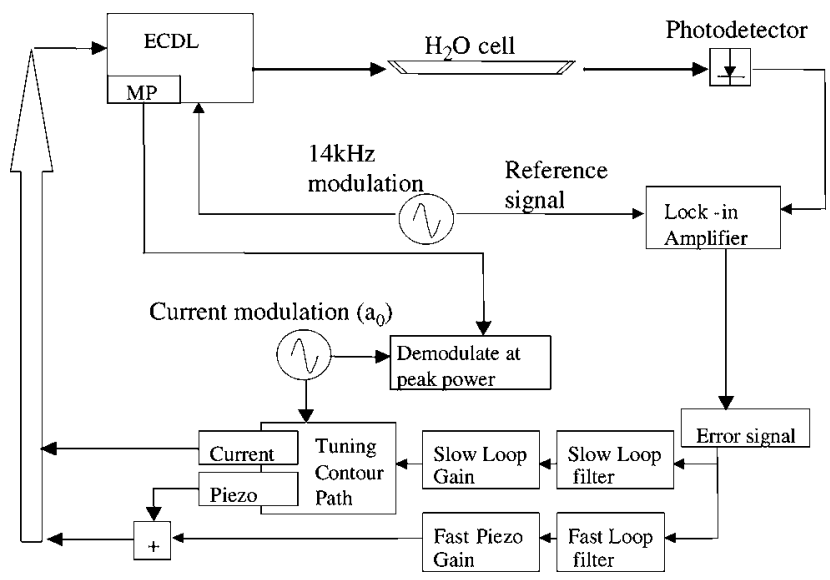

Fig. 3 Experimental setup used for the frequency stabilization of the ECDL with the dual feedback locking loop and mode referencing step illustrated. The modulation of the current $\left(a_{0}\right.$ term of the polynomial fit) of the wavelength tuning contour path, along with the internal monitor photodiode (MP) of the ECDL are also included.

erate an error signal between a reference frequency to which the laser output locks and the actual emission frequency of the device. In our case, the output of the ECDL was passed through a 1-m-long cell containing water vapor at a constant pressure of $25 \mathrm{mbar}$, and the resulting absorption signal was detected with a photodetector. A WMS scheme was implemented with the PZT being modulated at approximately $14 \mathrm{kHz}$ and a lock-in amplifier was used to process the detected signals. The modulation depth was set so that a maximum peak to peak amplitude on the $1 f$ demodulated signal was achieved. Modulating the external grating is preferred, as it helps reduce any amplitudemodulated component that is normally present with injection current modulation.

The frequency stabilization of the ECDL was controlled with a dual-feedback locking loop, which helped improve the overall performance and accuracy of the device. The feedback loop for the control of the ECDL is composed of two parts, a slow loop that tunes along the wavelength tuning contour with a combination of injection current and grating adjustment, and a second faster loop that controls the grating only. Since the fast loop drives only the grating piezoactuator, and not the laser current, there is no thermal interaction present so that the fast loop can operate at shorter timescales (milliseconds) in comparison to the slow loop. To demonstrate this scheme both parts of the feedback system were implemented as simple filtered proportional controllers, low pass for the slow branch and bandpass for the fast branch. For the slow branch, the low-pass filter cutoff and the proportional gain term are chosen to avoid any oscillatory behavior in the laser output frequency and to obtain critically damped behavior in the response to a step in laser current. ${ }^{16}$ The high-frequency side of the bandpass response of the fast branch was similarly determined, while the low-frequency side (within the slow loop response) ensures that the low-frequency components are handled by the slow loop along the tuning contour. Figure 3 shows the experimental setup for the frequency stabilization of an ECDL using a reference gas cell. A schematic description of the dual-feedback loop and the mode- 


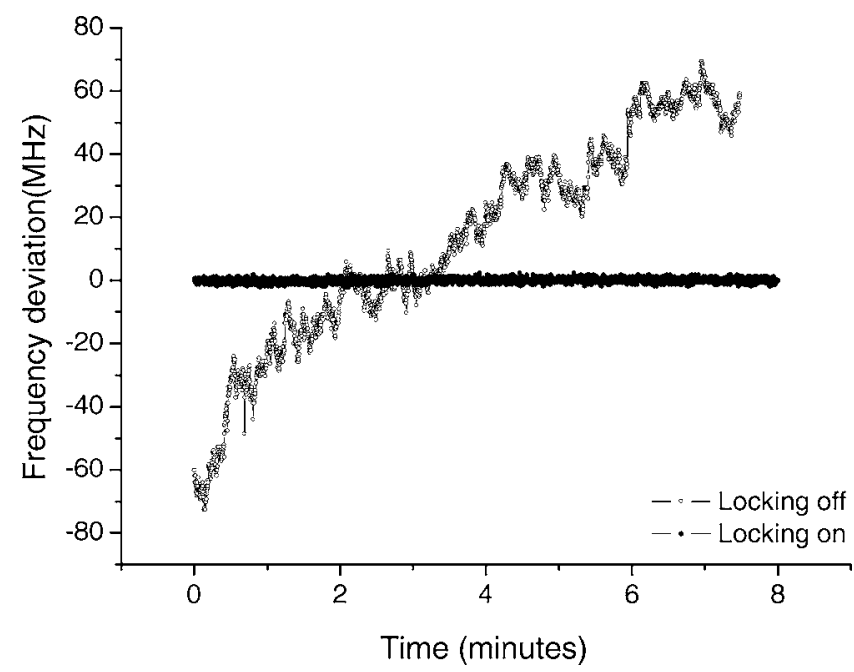

Fig. 4 Frequency stabilization performance of the ECDL with the dual-feedback locking switched on and off. The accuracy of the ECDL emission frequency in this case is approximately $\pm 1.5 \mathrm{MHz}$.

referencing step are also included in the figure. The result of the frequency stabilization of the ECDL output using the dual-feedback loop approach, by locking to the zero crossing of the $1 f$ harmonic of the $\mathrm{H}_{2} \mathrm{O}$ absorption line at $935.684 \mathrm{~nm}$, is presented in Fig. 4. The graph shows a comparison for the two cases where the locking is switched on and switched off for a period of $8 \mathrm{~min}$. The drift in frequency of the ECDL output when the locking is turned off is due to temperature effects and the free-running instability of the device. The overall accuracy of the frequency stabilized ECDL output to the absorption line center was measured to be less than $\pm 1.5 \mathrm{MHz}$.

\section{Mode Referencing}

As previously discussed, the necessity for a modereferencing step to control the emission frequency of the stabilized ECDL source emerged as a direct result of problems faced due to thermal and mechanical instability of the device and a consequential change in the contour mode plane. The contour mode plane determines the values of the injection current/PZT voltage combination, which provide the tuning path for continuous mode-hop-free wavelength tuning. One method that was initially considered for the mode-referencing step involved turning off the frequencylocking procedure, fixing the PZT voltage, and then tuning the laser output with injection current only, across the lasing mode. The range of the current tuning was set large enough so that the mode boundaries on either side of the lasing mode were crossed. Then by differentiating the resulting power change across the mode boundaries, recorded with the internal monitor photodiode of the device, the center of the lasing mode was acquired, the tuning path of the current/PZT voltage was realigned to the center of the lasing mode, and the frequency locking was subsequently switched back on. Obviously this worked well to reference the position of the lasing mode, but the major disadvantage of this method is a loss in frequency stabilization of the ECDL output with the locking switched off between scans, which ultimately would lead to a total failure of the frequency-locking process. In essence, with this method, the frequency locking must be periodically turned off to determine if there is any drift in operational characteristics that must be corrected to ensure continuation of the frequency locking

Our technique performs similarly, except with the advantage that the wavelength is constantly locked to the desired set point throughout the mode referencing, and the frequency stabilization scheme also remains in constant operation. Again, the main objective of the referencing is to align the wavelength-tuning path to the center of the lasing mode of interest so as to avoid any mode hops or reduced SMSR, which occur near the mode boundary positions. In our case, this is achieved by first locking the ECDL output to the absorption line of interest, and then, as the frequency stabilization is in operation, a modulation is applied to the current of the wavelength-tuning path. The tuning contour path is determined by a second-order polynomial fit, which selects the best fit of values of injection current/PZT voltage combination so that a well-defined wavelength-tuning contour path is achieved. The mode-referencing procedure enables the laser wavelength to remain constant because the $a_{0}$ term is in a perpendicular direction to the wavelengthtuning path. This step is indicated in Fig. 5, where the dashed line indicates the injection current/PZT voltage combination, which determines the wavelength-tuning path, and the arrow, which describes the modulation in the perpendicular direction to the wavelength-tuning path. A more detailed scan of the variation in output power of the ECDL, measured with the internal monitor photodiode across the mode, is shown in Fig. 6.

With this change in power, it was possible to set the amplitude of the modulation on the $a_{0}$ term large enough so that a value for peak power, corresponding to the center of the lasing mode, was measurable. Then it was simply a matter of setting up another feedback loop so that the wavelength-tuning path could be locked to the peak power of the lasing mode and, hence, locked to the center of the lasing mode. As the modulation on the $a_{0}$ term of the polynomial is in the direction perpendicular to the direction of continuous wavelength tuning, the locking frequency is maintained at the desired wavelength. The basis on which the emission wavelength of the ECDL can be maintained at the same value throughout the mode-referencing operation is explained clearly with reference to Fig. 5. The graph shows the recorded photodiode power of the detected laser radiation as a function of injection current and PZT voltage over a certain range, after passing through the 1-m gas cell containing water vapor at 25 mbar. From the trace, the positions of the various water absorption lines are identified by the points of reduced photodiode signal on the contour mode map. Note that the level of absorption is constant across the different modes. This confirms that the wavelength, in the direction perpendicular to the direction of the wavelength-tuning path, is constant so that the ECDL output can remain frequency stabilized to the desired wavelength $\left(\mathrm{H}_{2} \mathrm{O}\right.$ line at $\left.935.684 \mathrm{~nm}\right)$ throughout the mode referencing operation.

\section{Results}

To demonstrate the performance of the mode-referencing step a series of deliberate temperature changes to the ECDL 


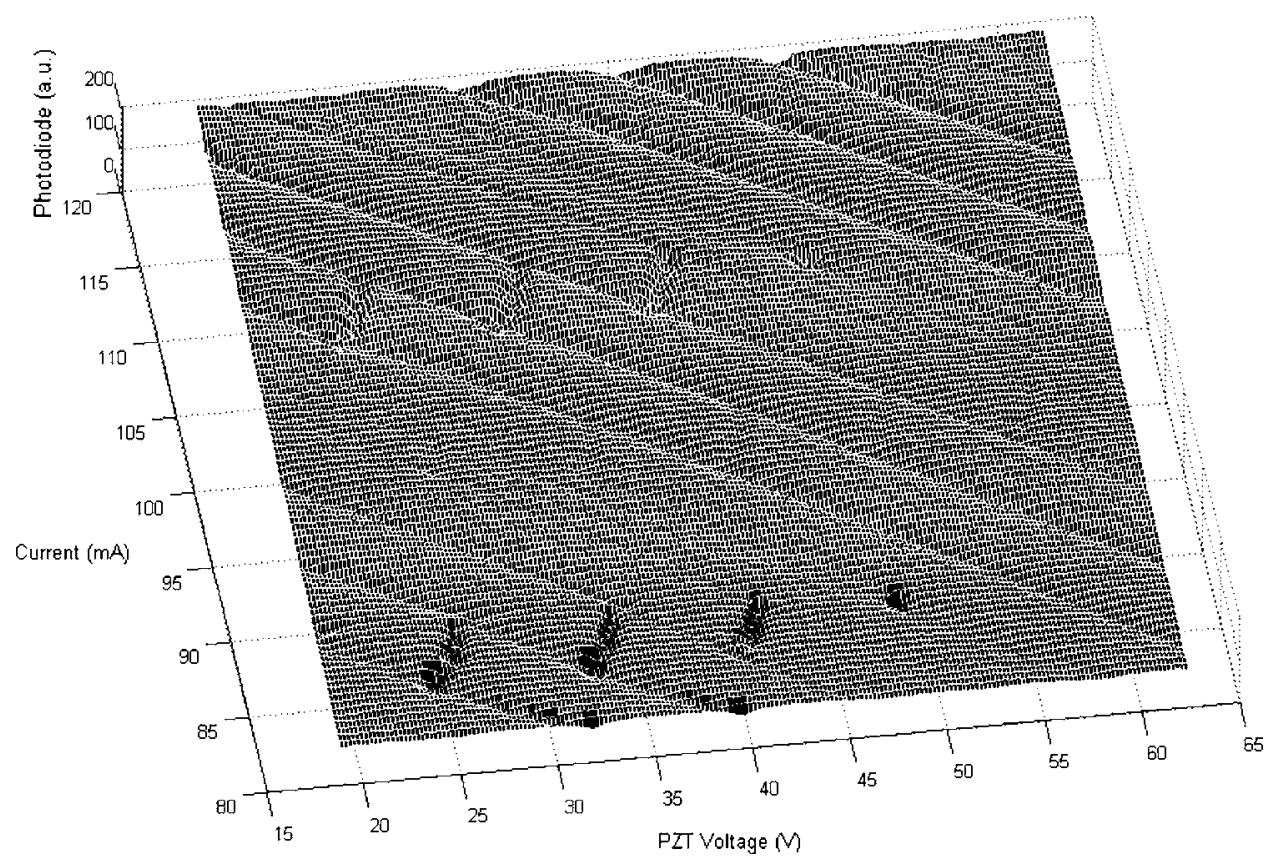

Fig. 5 Mode profile of ECDL emission measured with the detection through a water vapor cell at $24^{\circ} \mathrm{C}$ from 80 to $120 \mathrm{~mA}$ and 18 to $62 \mathrm{~V}$ as the current and PZT voltage are scanned. The points on the mode plane where water absorption lines are located are also clearly visible from the dips in the mode map, which correspond to a reduction in transmitted signal onto the photodiode.

heat sink were executed while the laser output was frequency stabilized. Figure 7 shows the output frequency of the ECDL while the device is frequency stabilized using the dual-feedback loop with the mode referencing switched on, as the heat sink temperature is varied from 23 to $24^{\circ} \mathrm{C}$ in $0.10^{\circ} \mathrm{C}$ steps by simply adjusting the temperature controller. It is clear that the output frequency of the stabilized ECDL does not vary by more that $5 \mathrm{MHz}$ for the duration (30 min) of the test. The change in temperature to the heat sink of the device essentially causes the contour mode profile to change, and this requires a change in the operating points of the current and PZT voltage if frequency locking

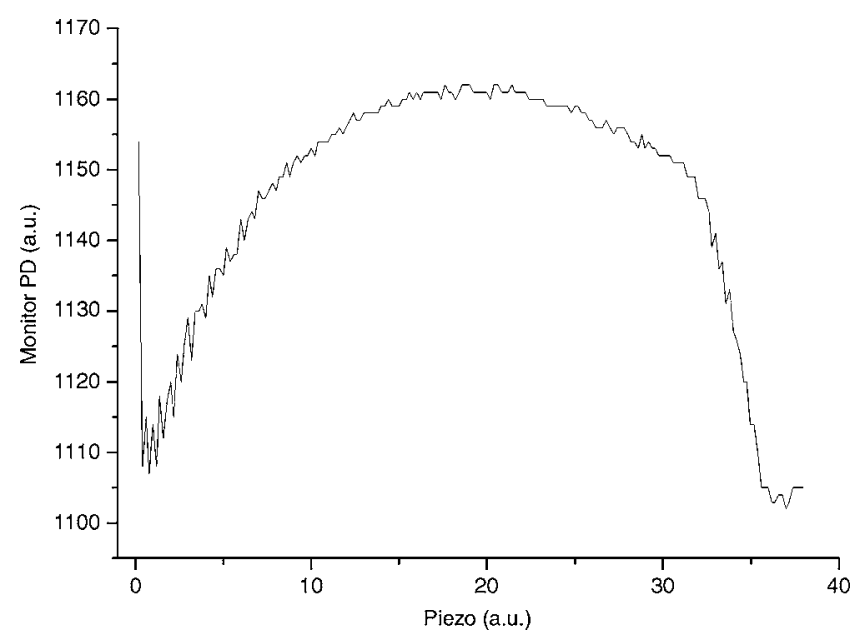

Fig. 6 Recorded MP showing the variation of the output power across a particular lasing mode. The current is kept constant and the PZT voltage is varied at constant temperature of $24^{\circ} \mathrm{C}$. to the desired frequency is to be maintained. The modereferencing tracks the change in the mode profile position with temperature, and then with the dual-feedback locking loop, the necessary changes to the injection current and PZT voltage are made. Figure 8 shows the variation in the current and PZT voltage for the duration of the test as the temperature is changed in $0.1^{\circ} \mathrm{C}$ increments for a total of $1{ }^{\circ} \mathrm{C}$. With the increase in temperature from 23 to $24^{\circ} \mathrm{C}$ it is clear that this results in an increase in PZT voltage from 28.4 to $35.0 \mathrm{~V}$ and a decrease in injection current from 110.5 to $107.3 \mathrm{~mA}$. It is also possible to see in the figure

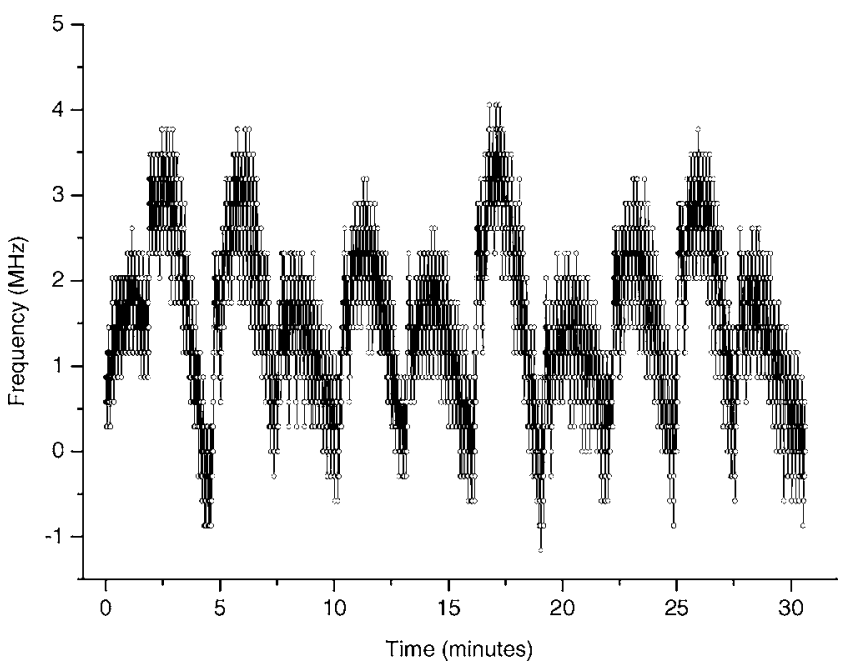

Fig. 7 Measured frequency drift of the ECDL output as the temperature is changed from 23 to $24^{\circ} \mathrm{C}$, in $0.1^{\circ} \mathrm{C}$ steps over a 60 -min period with the mode-referencing technique enabled. 


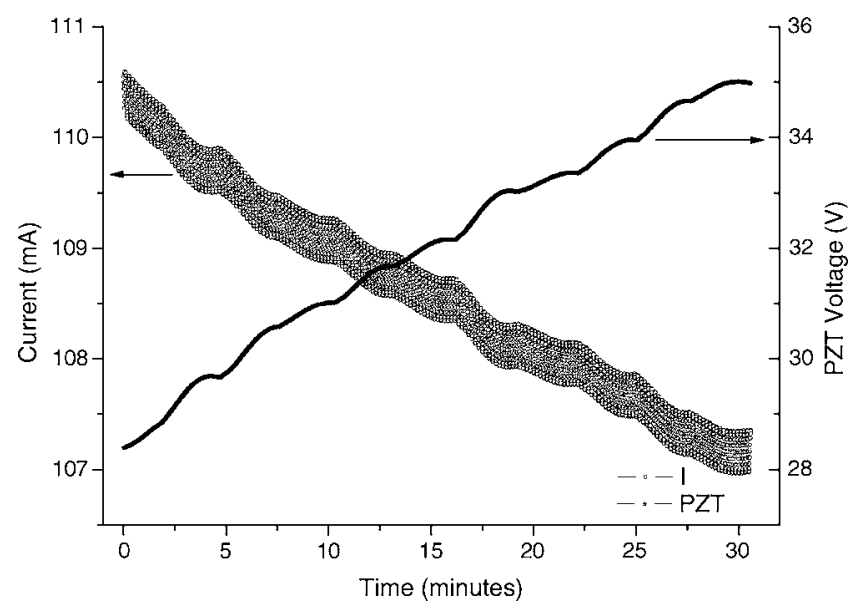

Fig. 8 Injection current and PZT voltage variation as the mode referencing step is in operation for the induced changes in the heat sink temperature of the ECDL.

the effect of the modulation on the $a_{0}$ term of the wavelength tuning path, clearly visible in the constant variation of the injection current. Finally, the trace in Fig. 9 shows the output power of the ECDL, recorded with the internal MP, as the temperature changes are made. An overall reduction in output power occurs as the mode referencing causes a change to both the current and PZT voltage while constantly maintaining frequency stabilization to the desired wavelength of $935.684 \mathrm{~nm}$.

\section{Conclusions}

The use of a novel mode-referencing technique was introduced, and was clearly shown to aid in the frequency stabilization of the output of an ECDL in conjunction with a dual-feedback locking loop. The addition of the modereferencing step greatly improved the operation and reliability of the frequency stabilization system that controls the frequency output of the laser. Its performance was

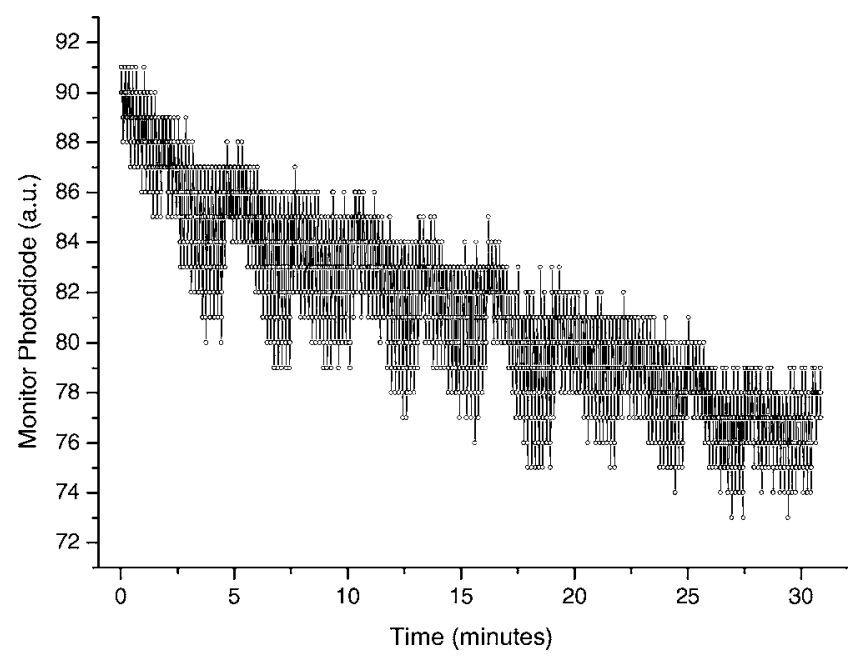

Fig. 9 Internal MP of ECDL showing a reduction in output power as the frequency locking and the mode referencing track the induced temperature changes. tested by frequency locking the ECDL and inducing a series of deliberate $0.1^{\circ} \mathrm{C}$ temperature steps from 23 to $24^{\circ} \mathrm{C}$ to the device heat sink without any failure of the locking system. The laser output frequency was maintained to within $5 \mathrm{MHz}$ of the $\mathrm{H}_{2} \mathrm{O}$ absorption line at $935.684 \mathrm{~nm}$ for the 30-min duration of the test. Therefore, with the addition of this mode-referencing technique to the frequency locking setup it is possible to operate the ECDL without having to repeat measurements of the mode contour profile, and thus maintain frequency stabilization. The essence of the technique is that it enables the frequency locking to continue uninterrupted. This contrasts with other schemes where the frequency locking must be periodically turned off to determine if there is any drift in operational characteristics that must be corrected to ensure continuation of the frequency locking.

\section{References}

1. Y. Sakai, I. Yokohama, T. Kominato, and S. Sudo, "Frequency stabilization of laser diode using a frequency-locked ring resonator to acetylene gas absorption lines," IEEE Photonics Technol. Lett. 3(10), 868-870 (1991).

2. T. Sato, M. Niikuni, S. Sato, and M. Shimba, "Frequency stabilisation of a semiconductor laser using Rb- $\mathrm{D}_{1}$ and $\mathrm{D}_{2}$ absorption lines," Electron. Lett. 24(7), 429-431 (1988).

3. A. Sollberger, A. Heinamaki, and H. Melchior, "Frequency stabilization of semiconductor lasers for applications in coherent communication systems," J. Lightwave Technol. 5(4), 485-491 (1987).

4. C. R. Prasad, V. A. Fromzel, J. S. Smucz, I. H. Hwang, and W. E. Hasselbrack, "A diode-pumped Cr:LiSAF laser for UAV-based water vapor differential absorption lidar (DIAL)," in Proc. Geoscience and Remote Sensing Symp., IGARSS 2000, Vol. 4, pp. 1465-1467, IEEE (2000).

5. V. Jayaraman, Z. M. Chuang, and L. A. Coldren, "Theory, design, and performance of extended tuning range semiconductor lasers with sampled gratings," IEEE J. Quantum Electron. 29(6), 1824-1834 (1993).

6. M. W. Fleming and A. Mooradian, "Spectral characteristics of external-cavity controlled semiconductor lasers," IEEE J. Quantum Electron. QE-17, 44-59 (1981).

7. R. Wyatt and W. J. Delvin, " $10 \mathrm{kHz}$ linewidth $1.5 \mu \mathrm{m}$ InGaAsP external cavity laser with $55 \mathrm{~nm}$ tuning range," Electron. Lett. 19, 110112 (1983).

8. B. Boggs, C. Greiner, T. Wang, H. Lin, and T. W. Mossberg, "Simple high-coherence rapidly tunable external-cavity diode laser," Opt Lett. 23, 1906 (1998).

9. G. Sarlet, G. Morthier, and R. Baets, "Control of widely tunable SSG-DBR lasers for dense wavelength division multiplexing," $J$. Lightwave Technol. 18(8), 1128-1138 (2000).

10. F. Delorme, G. Terol, H. de Bailliencourt, S. Grosmaire, and P. Devoldere, "Long-term wavelength stability of $1.55-\mu \mathrm{m}$ tunable distributed Bragg reflector lasers," IEEE J. Sel. Top. Quantum Electron. 5(3), 480-486 (1999).

11. S. L. Woodward, P. Parayanthal, and U. Koren, "The effects of aging on the Bragg section of a DBR laser," IEEE Photonics Technol. Lett. 5(7), 750-752 (1993)

12. S. A Al-Chalabi, J. Mellis, M. Hollier, K. H. Cameron, R. Wyatt, J. E. Regnault, W. J. Devlin, and M. C. Brain, "Temperature and mechanical vibration characteristics of a miniature long external cavity semiconductor laser," Electron. Lett. 26, 1159-1160 (1990).

13. H. Ishii, F. Kano, Y. Yoshikuni, and H. Yasaka, "Mode stabilization method for superstructure-grating DBR lasers," J. Lightwave Technol. 16, 433-442 (1998).

14. D. S. Bomse, A. C. Stanton, and J. A. Silver, "Frequency-modulation and wavelength modulation spectroscopies-comparison of experimental methods using a lead-salt diode-laser," Appl. Opt. 31, 718 731 (1992).

15. M. W. Fleming and A. Mooradian, "Spectral characteristics of external-cavity controlled semiconductor lasers," IEEE J. Quantum Electron. 17, 44-59 (1981).

16. J. R. Leigh, Control Theory, 2nd ed., Chap. 3, Institution of Electrica Engineers (2004).

Biographies and photographs of authors not available. 